\title{
A New Approach to Model Reference Control for a Class of Arbitrarily Fast Time-varying Unknown Plants*
}

\author{
CHIANG-JU CHIEN $\dagger$ and LI-CHEN FU $\ddagger$
}

Key Words-Model reference control; linear fast time-varying; variable structure design.

\begin{abstract}
In this note, a new standard model reference control (NSMRC) to arbitrarily fast linear single-input single-output time-varying plants with relative degree one is presented. A modified version of standard model reference control (SMRC) structure is developed which incorporates the concept of variable structure design and null adaptation process for fast time-varying plants. It is shown that the output error will converge to zero in finite time without any restriction on the time-varying rate of the plant parameters while all signals inside the closed-loop system remain uniformly bounded so long as some design parameters are chosen properly (large). Superior transient responses as well as convergence properties can be observed from the simulation results provided.
\end{abstract}

\section{Introduction}

IN THE FIELD of research on controlling linear time-varying unknown systems, adaptive approaches are usually taken under some assumptions on the plant parameter variations (suitable time-varying models) such as in Gomart and Caines (1986), Zheng (1987), Middleton and Goodwin (1988), Tsakalis and Ioannou $(1987,1989,1990)$. No matter what class of models is chosen, the restriction on time-varying parameters plays an important role in the study of these problems. In particular, the time-varying rate of plant parameters becomes a crucial assumption if their timevarying structure is not known in advance.

In this note, in contrast with the usual adaptive approaches, we propose a nonadaptive new standard model reference control (NSMRC) method to solve the problem for arbitrarily fast time-varying plants of relative degree one with much less-restriction on the unknown parameters. Our idea is originated from the new model reference control (NMRC) scheme proposed by Tsakalis and Ioannou (1989) where a "matching condition" can be achieved between the compensated closed-loop plant and time-invariant reference model when plant coefficients are completely known. By using the results described above, together with the concept of variable structure design, we get a NSMRC scheme which can be used to control the arbitrarily fast time-varying plants with unknown parameters.

\section{A matching condition for NSMRC with time-varying} plants

Consider a single-input-single-output (SISO) linear time-varying plant, which can be described by the following

${ }^{*}$ Received 15 October 1990; recommended for publication in revised form by Editor W. S. Levine.

$\dagger$ Department of Electrical Engineering, National Taiwan University, Taipei, Taiwan, Republic of China. Author to whom all correspondence should be addressed.

$\ddagger$ Departments of Electrical Engineering, and Computer Science and Information Engineering, National Taiwan University, Taipei, Taiwan, Republic of China.

This research is supported by National Science Council under the grant NSC 79-0404-E-002-03. form of differential equations:

$$
\begin{aligned}
D_{p}(s, \rho)\left[x_{p}(t)\right] & =u_{p}(t) ; \quad X_{p}(0)=X_{p 0} \\
y_{p}(t) & =k_{p}(\rho) N_{p}(s, \rho)\left[x_{p}(t)\right]
\end{aligned}
$$

where $x_{p} \in R^{n}$ is an internal state; $u_{p}, y_{p} \in R$ are input and output of the plant, respectively; $D_{p}(s, \rho), N_{p}(s, \rho)$ are monic linear time-varying PDOs with degree $n, m$, respectively (see Tsakalis and Ioannou, 1987, 1989 for detail); $k_{P}(\rho) \neq$ $0, \forall \rho \geq 0$ is the high frequency gain and $X_{p}(0)=$ $\left[x_{p}(0), \ldots, x_{p}^{(n-1)}(0)\right]^{\top}$ is the initial condition.

It should be noted that all the plant parameters (coefficients of the PDOs) are uniformly bounded smooth functions of $\rho$, where $\rho=\mu t$ is the parameter time scale with $\mu \geq 0$, i.e. $\mu$ is the ratio of the time scale of plant parameters versus that of plant states.

The control objective is to design a control law $u_{p}(t)$ such that the output $y_{p}(t)$ of the plant tracks the output $y_{m}(t)$ of a linear time-invariant reference model

$$
y_{m}=W_{m}(s) r=k_{m} D_{m}^{-1}(s) r
$$

where $D_{m}(s)$ is a monic Hurwitz polynomial, $k_{m}>0$, and $r(t)$ is a uniformly bounded reference input signal. To make the problem more tractable, several assumptions on the plant and reference model are made: (S1) $n, m$ are known. (S2) $D_{p}(s, \rho), N_{p}(s, \rho)$ are strongly right coprime PDOs with unknown time-varying coefficients. (S3) The sign of $k_{p}(\rho)$ is constant with respect to $\rho$ and is known a priori. Without loss of generality, we will assume that $k_{p}(\rho)>0, \forall \rho \geq 0$ and the range of $k_{p}(\rho)$ is a subset of a closed interval on the real axis that does not contain 0 . (S4) $N_{p}^{-1}(s, \rho)$ is an exponentially stable PIO. (S5) $D_{m}(s)$ is designed so that degree of $D_{m}(s)$ is $n-m$.

In the SMRC structure, auxiliary vectors $\bar{w}_{1}, \bar{w}_{2}$ are generated according to the following:

$$
\dot{w}_{1}=\Lambda \bar{w}_{1}+l u_{p}, \quad \dot{w}_{2}=\Lambda \bar{w}_{2}+l y_{p}
$$

where $(\Lambda, l)$ is a controllable pair with $\Lambda \in R^{n-1 \times n-1}$ being a stable matrix. Let $\bar{w}=\left[\bar{w}_{1}^{\top}, \bar{w}_{2}^{\top}, y_{p}, r\right]^{\top}$ and $\bar{\theta}=$ $\left[\bar{\theta}_{1}^{\top}, \bar{\theta}_{2}^{\top}, \theta_{3}, c_{o}\right]^{\top}$. The following lemma will state a condition on the input $u_{p}$ so that an exact match between the closed-loop plant and the reference model is obtained from the point of view of I/O operators for our NSMRC structure.

Lemma 1 . There exists a vector $\bar{\theta}^{*}(\rho)$ such that, if $u_{p}(t)$ is designed as:

$$
\begin{aligned}
u_{p}= & \left\{c_{o}^{*}(\rho) r+\bar{\theta}_{1}^{* \top}(\rho) \bar{w}_{1}+\bar{\theta}_{2}^{* \top}(\rho) \bar{w}_{2}+\theta_{3}^{*}(\rho) y_{p}\right\} \\
& +\left\{\mu d^{-1}(s) X_{1}^{*}(s, \rho) d^{-1}(s) u_{p}\right. \\
& \left.+\mu d^{-1}(s) X_{2}^{*}(s, \rho) d^{-1}(s) y_{p}\right\} \\
= & \left\{\bar{\theta}^{* \top} \bar{w}+\mu \eta_{1}+\mu \eta_{2}\right\}
\end{aligned}
$$

where $d(s)=\operatorname{det}(s I-\Lambda)$ and $X_{i}^{*}(s, \rho)$ is a PDO whose coefficients depend on the derivatives of $\bar{\theta}_{i}^{*}(\rho)$, for $i=1,2$, 
the I/O operator of the closed-loop plant $G_{c}(s, t): r \rightarrow y_{p}$ is equal to the time-invariant reference model

Proof. See Fu and Chien (1990).

3. An NSMRC for an unknown time-varying plant

In this section, the new standard model reference control scheme for a fast time-varying plant with relative degrec one is presented.

3.1. Error model. When plant parameters are known and under the matching condition for our NSMRC scheme, the plant output can be expressed, from an operator point of view, as

$$
y_{p}=W_{m}(s) r .
$$

If the time-varying parameters of the plant are unknown, equation (2.4) and, hence, (3.1) will not be achievable so that (3.1) has to be modified with extra input signals besides the exogenous input $r$. Define $\bar{\phi}_{i}=\bar{\theta}_{i}-\bar{\theta}_{i}^{*}(\rho), i=1,2$, $\bar{\phi}_{3}=\theta_{3}-\theta_{3}^{*}(\rho), \bar{\phi}_{4}=c_{o}-c_{o}^{*}(\rho)$ and $\bar{\phi}=\left[\bar{\phi}_{1}^{\top}, \bar{\phi}_{2}^{\top}, \bar{\phi}_{3}, \bar{\phi}_{4}\right]^{\top}$ so that it can be seen that the plant input $u_{p}$ originally given in (2.4) for matching condition will now become,

$$
u_{p}=\left(\bar{\theta}^{* \top} \bar{w}+\mu \eta_{1}+\mu \eta_{2}\right)+\left(\bar{\phi}^{\top} \bar{w}-\mu \eta_{1}-\mu \eta_{2}\right) \equiv u_{p 1}+u_{p, 2} .
$$

It is clear that the first term $u_{p 1}$ satisfies the matching condition for plant input in the NSMRC structure. So, with plant parameters being unknown, the output $y_{p}$, should be as follows:

$$
y_{p}=W_{m}(s)\left(c_{o}^{*}(\rho)^{-1}\left(\bar{\phi}^{\top} \bar{w}-\mu \eta_{1}-\mu \eta_{2}\right)+r\right) .
$$

Define output error $e_{o}$ by $e_{o}=y_{p}-y_{m}$ and apply an additional switching signal $v_{p}$ (Fu, 1989) to be defined later into the plant input channel, we can then get the following output error equation,

$$
\boldsymbol{e}_{o}=W_{m}(s) c_{o}^{*}(\rho)^{-1}\left(\bar{\phi}^{\top} \bar{w}-\mu \eta_{1}-\mu \eta_{2}+v_{p}\right) .
$$

Now, by any minimal realization $\left(A_{m}, b_{m}, c_{m}\right)$ for $W_{m}(s)$. the error model can be written as:

$$
\begin{aligned}
\dot{e} & =A_{m} e+b_{m} c_{o}^{*}(\rho)^{-1}\left(\bar{\phi}^{\top} \bar{w}-\mu \eta_{1}-\mu \eta_{2}+v_{p}\right) \\
e_{o} & =c_{m}^{\top} e .
\end{aligned}
$$

3.2. Controller design for fast time-varying plants. In this subsection, we focus on an MRAC problem for fast time-varying plants using the formulation obtained previously. Resolution of the problem usually involves an input control law along with a parameter adaptation law. But here, a different approach is assumed which forsakes the adaptation process and fix $\bar{\theta}$ at a constant zero vector, i.e. $\bar{\theta} \equiv \mathbf{0}$ so that only a bound on $\left\|\bar{\theta}^{*}\right\|$ is used later in our input synthesis to compensate for the lack of knowledge of $\bar{\theta}^{*}$. The following theorem will state our main result on the NSMRC for fast time-varying plants.

Theorem 1. Consider the error system (3.5) with a switching input $v_{p}$ defined as

$$
v_{p}=-\operatorname{sgn}\left(e_{p}\right)\left(\beta_{0}\left\|\bar{w}_{l}\right\|_{x}+\beta_{1}\right)
$$

where $\left\|\bar{w}_{t}\right\|_{x}=\sup _{r=1}\|\bar{w}(\tau)\|$ and let $\bar{\theta}=0$. Then for any time-varying rate of plant parameters, therc exist $\beta_{0}^{*}, \beta_{1}^{*}>0$ such that all signals inside the closed-loop system are uniformly bounded and the tracking error $e_{\text {, will converge to }}$ zero in finite time so long as $\beta_{0} \in\left(\beta_{0}^{*}, \infty\right)$ and $\beta_{1} \in\left(\beta_{1}^{*}, \infty\right)$.

\section{Proof. See Appendix A.}

Remark 1. In this NSMRC scheme, no parameter adaptation is used mainly because that any finite speed adaptation can not obtain an effective estimation for fast unstructured parameter variations. Another reason is that large amount of computation can be dropped and, hence, the complexity of the overall control scheme is much reduced. This feature, however, can not be shared with a similar scheme but using NMRC structure since fixing $\theta$, say, at zero will not provide any meaningful regressor signals ready to compensate for unknown dynamics of the closed-loop plant.

Remark 2. Although the NMRC scheme can also deal with fast time-varying plants, some a priori knowledge about the structure of the fast parameter variations has to be assumed. In the case where unstructured parameter variations are encountered (as has been discussed throughout this note), the slowly time-varying assumption on the plant parameters is still crucial to the stability property of the closed-loop system there. But it is noteworthy that for any time-varying rate $\mu_{1} \geq 0, \beta_{0}^{*}$ and $\beta_{1}^{*}$ defined in (A.16) (Appendix A) will always suffice in Theorem 1. This, in turn, implies that the NSMRC scheme proposed here will have the desired property for any time-varying plant so long as its time-varying rate $\mu \in\left[0, \mu_{1}\right]$. However, the cost of controlling arbitrarily fast time-varying plants (arbitrarily large value of $\mu_{1}$ ) is the increase of control parameters $\beta_{0}^{*}$ and $\beta_{1}^{*}$ and, hence, the increase of control force $v_{p}$.

\section{Simulation}

In this section, we give a simulation example to demonstrate the performance of the NSMRC scheme. The plant in the present example is the same as the one in that presented in Tsakalis and Ioannou (1987), which is described as follows.

$$
\begin{gathered}
x^{(2)}+(2 \sin (\mu t)-5 \exp (-\mu t)) x^{(1)} \\
+\left(\sin ^{2}(\mu t)-5 \sin (\mu t) \exp (-\mu t)\right) x=u_{p} \\
y_{p}=x^{(1)}+(3+\sin (\mu t)) x .
\end{gathered}
$$

The reference model and its input are chosen as $\frac{1}{(s+1)}$ and $10 \sin (t)$, respectively. Figure 1 is the simulation for the fast time-varying $(\mu=25)$ plants with initial conditions $x(0)=1.5, x^{(1)}(0)=1$. The control parameters are chosen as $\beta_{0}=30, \beta_{1}=15$. From the simulation results, we
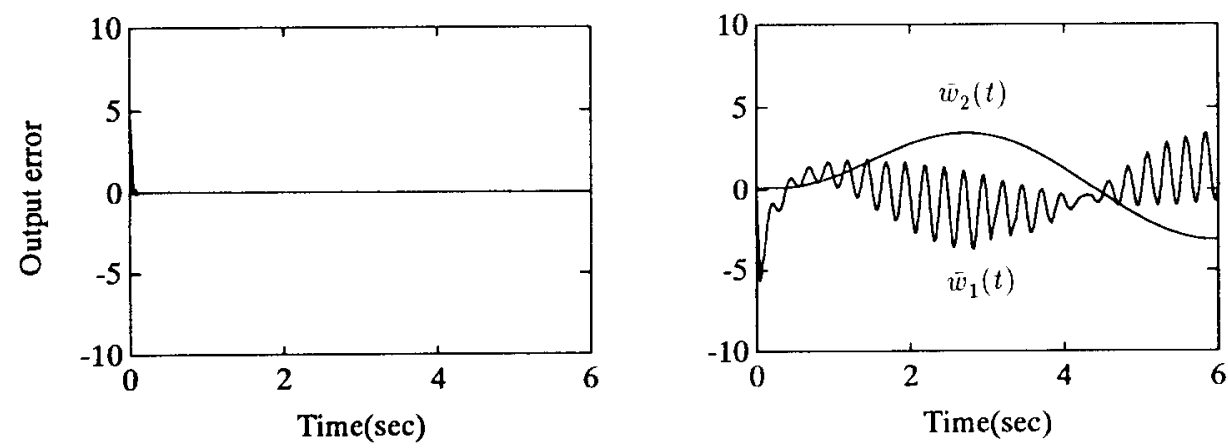

FIG. 1. Finite time output tracking and bounded signal response for plant time scale $\mu=25$. 
can see that the perfect tracking property and good transient response are achieved.

\section{Conclusion}

In this note, a new standard model reference control (NSMRC) approach has been proposed to solve the typical MRAC problem for linear time-varying plants. The approach has first transformed the time-varying effect of plant parameters into some additional signals which, when injected to the input channel of the plant, facilitates an exact match in the SMRC structure from the point of view of I/O operators. The transformation enables us to develop a NSMRC structure which is especially suitable for incorporating the variable structure design concept whereby the output error converges to zero in finite time so long as the design parameters are chosen properly (large). In addition, all signals insided the closed-loop system will remain uniformly bounded.

\section{References}

Fu, L. C. (1989). A robust model reference adaptive control using variable-structure adaptation for a class of plants. Proc. 1989 ACC, 2291-2296.

Fu, L. C. and C. J. Chien (1990). A new approach for solving MRAC problem for fast time-varying unknown plants with relative degree one. Proc. $1990 A C C$, 1058-1063.

Gomart, O. and P. Caines (1986). On the extension of robust global adaptive control results to unstructured time-varying systems. IEEE Trans. Aut. Control, AC-31, 370-373.

Middleton, R. H. and G. C. Goodwin (1988). Adaptive control of time-varying linear system. IEEE Trans Aut. Control, AC-33, 150-155.

Narendra, K. S. and L. Valavani (1978). Stable adaptive controller design-direct control. IEEE Trans. Aut. Control, AC-23, 570-583.

Narendra, K. S. and A. M. Annaswamy (1988). Stable Adaptive Systems. Prentice-Hall, Englewood Cliffs, New Jersey.

Tsakalis, K. S. and P. A. Ioannou (1987). Adaptive control of linear time-varying plants. Automatica, 23, 459-468

Tsakalis, K. S. and P. A. Ioannou (1989). Adaptive control of linear time-varying plants: A new model reference controller structure. IEEE Trans. Aut. Control, AC-34, 1038-1046.

Tsakalis, K. S. and P. A. Ioannou (1990). A new indirect adaptive control scheme for time-varying plants. IEEE Trans. Aut. Control, AC-35, 697-705.

Zheng, L. (1987). Discrete-time adaptive control of deterministic time-varying plants. IEEE Trans. Aut. Control, AC-32, 444-447.

\section{Appendix A: Proof of Theorem 1}

Step 1. At first, we use a similar way to Narendra and Annaswamy (1988) to establish the relation between the growth rates of all signals in the closed-loop, assuming that all signals grow in an unbounded fashion.

Note that $N_{p}^{-1}(s, \rho)$ is an exponentially stable PIO and

$$
y_{p}=k_{p}(\rho) N_{p}(s, \rho) D_{p}^{-1}(s, \rho) u_{p}=k_{p}(\rho) N_{p}(s, \rho) D_{p}^{-1}(s, \rho) v_{p}
$$

(where $u_{p}=v_{p}$ since $\bar{\theta}=0$ ). Let $\ddot{v}_{p}=(s+a)\left(\frac{1}{s+a}\right) v_{p}$, where $a>0$ so that $\frac{1}{s+a}$ is a stable operator. It is easy to show that $v_{p}=\bar{v}_{p}$ for any initial condition and, hence,

$$
y_{p}=k_{p}(\rho) N_{p}(s, \rho) D_{p}^{-1}(s, \rho)(s+a)\left(\frac{1}{s+a}\right) v_{p}
$$

which implies that

$$
\frac{1}{s+a} D_{p}(s, \rho) N_{p}^{-1}(s, \rho) k_{p}^{-1}(\rho) y_{p}=\frac{1}{s+a} v_{p} \equiv v_{p 1}
$$

Since $\frac{1}{s+a} D_{p}(s, \rho) N_{p}^{-1}(s, \rho) k_{p}^{-1}(\rho)$ is a proper stable operator, we can conclude from Lemma 2.6 in Narendra and Annaswamy (1988) that

$$
\left|v_{p 1}\right| \leq k_{1}\left\|y_{p t}\right\|_{\infty}+k_{2}
$$

for some positive constants $k_{1}$ and $k_{2}$. Our purpose here is to show that $v_{p}$ cannot grow faster than $v_{p 1}$ which, together with (A.4), implies that $v_{p}$ (plant input) will be bounded by $y_{p}$ (plant output). However, to show that $v_{p}$ cannot grow faster than $v_{p 1}$ is the same case as Lemma 2.8 in Narendra and Annaswamy (1988) if $v_{p}$ satisfies the following requirement

$$
\left|v_{p}\left(t_{1}\right)\right| \geq c_{2}\left|v_{p}\left(t_{1}+T\right)\right|
$$

where $t_{1}$ and $t_{1}+T$ are the time defined as:

$$
\left[t_{1}, t_{1}+T\right] \subset \Omega=\left\{t|| v_{p}(t) \mid=\left\|v_{p t}\right\|_{\infty}\right\}
$$

and $c_{2}$ is a constant $\in(0,1)$. Now, let (A.1) be represented as the following state space realization,

$$
\dot{x}_{p}(t)=A_{p}(t) x_{p}(t)+b_{p}(t) v_{p}(t), \quad y_{p}(t)=c_{p}(t) x_{p}(t) .
$$

By the following augmented system,

$$
\frac{\mathrm{d}}{\mathrm{d} t}\left[\begin{array}{c}
\bar{w}_{1} \\
\bar{w}_{2} \\
x_{p}
\end{array}\right]=\left[\begin{array}{ccc}
\Lambda & 0 & 0 \\
0 & \Lambda & l c_{p}(t) \\
0 & 0 & A_{p}(t)
\end{array}\right]\left[\begin{array}{c}
\bar{w}_{1} \\
\bar{w}_{2} \\
x_{p}
\end{array}\right]+\left[\begin{array}{c}
l \\
0 \\
b_{p}(t)
\end{array}\right] v_{p}
$$

we can easily show that, for some positive constants $k_{3}, k_{4}$ and $\xi=\left[\bar{w}_{1}^{\top}, \bar{w}_{2}^{\top}, x_{p}^{\top}\right]^{\top}$,

$$
\|\dot{\xi}\| \leq k_{3}\|\xi\|+k_{4}\left|v_{p}\right| .
$$

Since $A_{p}(t), b_{p}(t)$ and $c_{p}(t)$ are uniformly bounded by hypothesis. Because $\left|v_{p}\right|=\beta_{0}\left\|\bar{w}_{t}\right\|_{\infty}+\beta_{1}$ and $\left|y_{p}\right| \leq$ $\left\|c_{p}\right\|\left\|x_{p}\right\|$, we can show that, for some $k_{5}, k_{6}>0$,

$$
\|\dot{\xi}\| \leq k_{5}\left\|\xi_{t}\right\|_{\infty}+k_{6}
$$

which implies $\xi$ and, hence, $v_{p}$ can grow at most exponentially fast and the requirement (A.5) is satisfied. Consequently, the fact that $v_{p}$ cannot grow faster than $y_{p}$ is then established.

Step 2. Construct a Lyapunov function $V(e)=\frac{1}{2} e^{\top} P e$ where $P=P^{\top}>0$. Then the time derivative of $V$ along the trajectories of (3.5) subject to $v_{p}$ can be computed as:

$$
\begin{aligned}
\dot{V}= & -e^{\top}\left(A_{m}^{\top} P+P A_{m}\right) e+e^{\top} P b_{m} c_{o}^{*}(\rho)^{-1} \\
& \times\left(-\bar{\theta}^{* \top} \bar{w}-\mu \eta_{1}-\mu \eta_{2}+v_{p}\right)
\end{aligned}
$$

where we use the fact $\bar{\phi}=-\bar{\theta}^{*}$. By the strictly positive real (SPR) property of $W_{m}(s)$, we can find a $Q=Q^{\dagger}>0$ such that $A_{m}^{\top} P+P A_{m}=-Q$ and $P b_{m}=c_{m}$. Furthermore, according to assumption (S3), we can conclude that $c_{o}^{*}(\rho)>0, \forall \rho \geq 0$. Then, $\dot{V}$ in (A.11) can be found as:

$$
\begin{aligned}
\dot{V}= & -e^{\top} Q e+e_{o} c_{o}^{*}(\rho)^{-1}\left\{-\bar{\theta}^{* \top} \bar{w}-\mu \eta_{1}-\mu \eta_{2}\right. \\
& \left.-\operatorname{sgn}\left(e_{o}\right)\left(\beta_{0}\left\|\bar{w}_{t}\right\|_{\infty}+\beta_{1}\right)\right\} \\
& \leq-q\|e\|^{2}-\left|e_{o}\right| c_{o}^{*}(\rho)^{-1}\left\{\beta_{0}\left\|\bar{w}_{t}\right\|_{\infty}+\beta_{1}\right. \\
& \left.-\left\|\bar{\theta}^{*}\right\|\left\|\bar{w}_{t}\right\|_{\infty}-\mu\left|\eta_{1}\right|-\mu\left|\eta_{2}\right|\right\}
\end{aligned}
$$

for some positive constant $q$. It is easily shown that $\operatorname{deg}\left(X_{i}^{*}(s, \rho)\right) \leq(n-1)+(n-1)-1=2 n-3$, which along with the fact that $\operatorname{deg}(d(s))=n-1$ implies $d^{-1}(s) X_{i}^{*}(s, \rho) d^{-1}(s)$ is a strictly proper stable operator (Tsakalis and Ioannou, 1989). Hence, we can show that

$$
\left|\eta_{1}\right| \leq \bar{M}_{1}\left\|u_{p t}\right\|_{\infty}+\bar{M}_{2}, \quad\left|\eta_{2}\right| \leq \bar{M}_{3}\left\|y_{p r}\right\|_{\infty}+\bar{M}_{4}
$$

for some positive constants $\bar{M}_{i}, i=1,2,3,4$. By the results derived in step 1 , we have

$$
\left|\eta_{1}\right| \leq M_{1}\left\|\bar{w}_{t}\right\|_{\infty}+M_{2}, \quad\left|\eta_{2}\right| \leq M_{3}\left\|\bar{w}_{f}\right\|_{\infty}+M_{4}
$$

for some positive constants $M_{i}, i=1,2,3,4$. Substitution of 
(A.14) into (A.12) then yields,

$$
\begin{aligned}
\dot{V} \leq & -q\|e\|^{2}-\left|e_{o}\right| c_{o}^{*}(\rho)^{-1}\left\{\left(\beta_{0}-\left\|\bar{\theta}^{*}\right\|-\mu M_{1}-\mu M_{3}\right) \|\left.\bar{w}_{1}\right|_{\infty}\right. \\
& \left.+\left(\beta_{1}-\mu M_{2}-\mu M_{4}\right)\right\} .
\end{aligned}
$$

If we choose the design parameters $\beta_{0}, \beta_{1}$ in such a way that

$$
\begin{aligned}
& \beta_{0}>\beta_{0}^{*}=\left\|\bar{\theta}^{*}\right\|+\mu M_{1}+\mu M_{3} \\
& \beta_{1}>\beta_{1}^{*}=\mu M_{2}+\mu M_{4}
\end{aligned}
$$

then $\dot{V} \leq-q\|e\|^{2}$ which concludes that $e$ and, hence, $e_{0}$ approach zero at least exponentially fast. Note that the zero-convergence of $e_{o}$ directly implies that the plant output $y_{p}\left(=e_{o}+y_{m}\right)$ is bounded, $\forall t \geq 0$. Also by the fact that $v_{p}$ cannot grow faster than $y_{p}$, the boundedness of all signals in the closed-loop is established.

Step 3. Finally, the property of finite time convergence on output error $e_{o}$ should be established. Observing the error model (3.5), with the condition $\bar{\phi}=-\bar{\theta}^{*}$, we have

$$
\begin{aligned}
\frac{1}{2} \frac{d e_{o}^{2}}{d t}= & e_{o} c_{m}^{\top} A_{m} e+e_{o} c_{m}^{\top} b_{m} c_{o}^{*}(\rho)^{-1} \\
& \times\left\{-\bar{\theta}^{* \top} \bar{w}-\mu \eta_{1}-\mu \eta_{2}+v_{p}\right\} \\
& \leq\left|e_{o}\right|\left\{k_{7}\|e\|-k_{8}\left[\left(\beta_{0}-\left\|\bar{\theta}^{*}\right\|-\mu M_{1}-\mu M_{3}\right)\right.\right. \\
& \left.\left.\times\left\|\bar{w}_{1}\right\|_{\infty}+\left(\beta_{1}-\mu M_{2}-\mu M_{4}\right)\right]\right\}
\end{aligned}
$$

where $k_{7}, k_{8}>0$ are some appropriate constants. Since $\|e(t)\|$ approaches zero at least exponentially fast and (A.16) is assumed, there exists a finite $T_{f} \geqslant 0$ such that

$\frac{1}{2} \frac{\mathrm{d} e_{o}^{2}}{\mathrm{~d} t} \leq-k_{9}\left|e_{o}\right|$ for all $t>T_{f}$ and for some $k_{9}>0$, which implies that the switching surface $e_{o}=0$ will be reached in finite time. This concludes our proof. 